\title{
TBS IMPROVES THE DETECTION OF SUBJECTS AT RISK OF FRACTURE IRRESPECTIVELY TO THE BMD STATUS: a Spanish population-based study.
}

\author{
C E T I R $\bigcirc$ GRUPMËOC \\ L. Del Rio, S. Di Gregorio, R. Winzenrieth(*) \\ CETIR Grup Mèdic, Barcelona. $\left({ }^{*}\right)$ Medimaps, Pessac, France
}

\section{INTRODUCTION}

Vertebral fractures produced by low-energy impacts, can be a consequence of a bone with low bone density and / or with altered structural quality. Today, the determination of bone mineral density - as assessed by DXA - can identify subjects at increased risk of fracture when density measurements are found below certain ranges.

The Trabecular Bone Score (TBS) is a texture parameter assessing pixel gray-level variations DXA images. TBS evaluation is derived from experimental variogram and linked to bone microarchitecture status.

The software for TBS computation (TBS iNsight, Medimpas, France) is installed directly on DXA machines, and TBS is automatically calculated consecutively to BMD measurement. A low TBS value is intuitively interpreted as a low quality of bone texture. TBS Principles

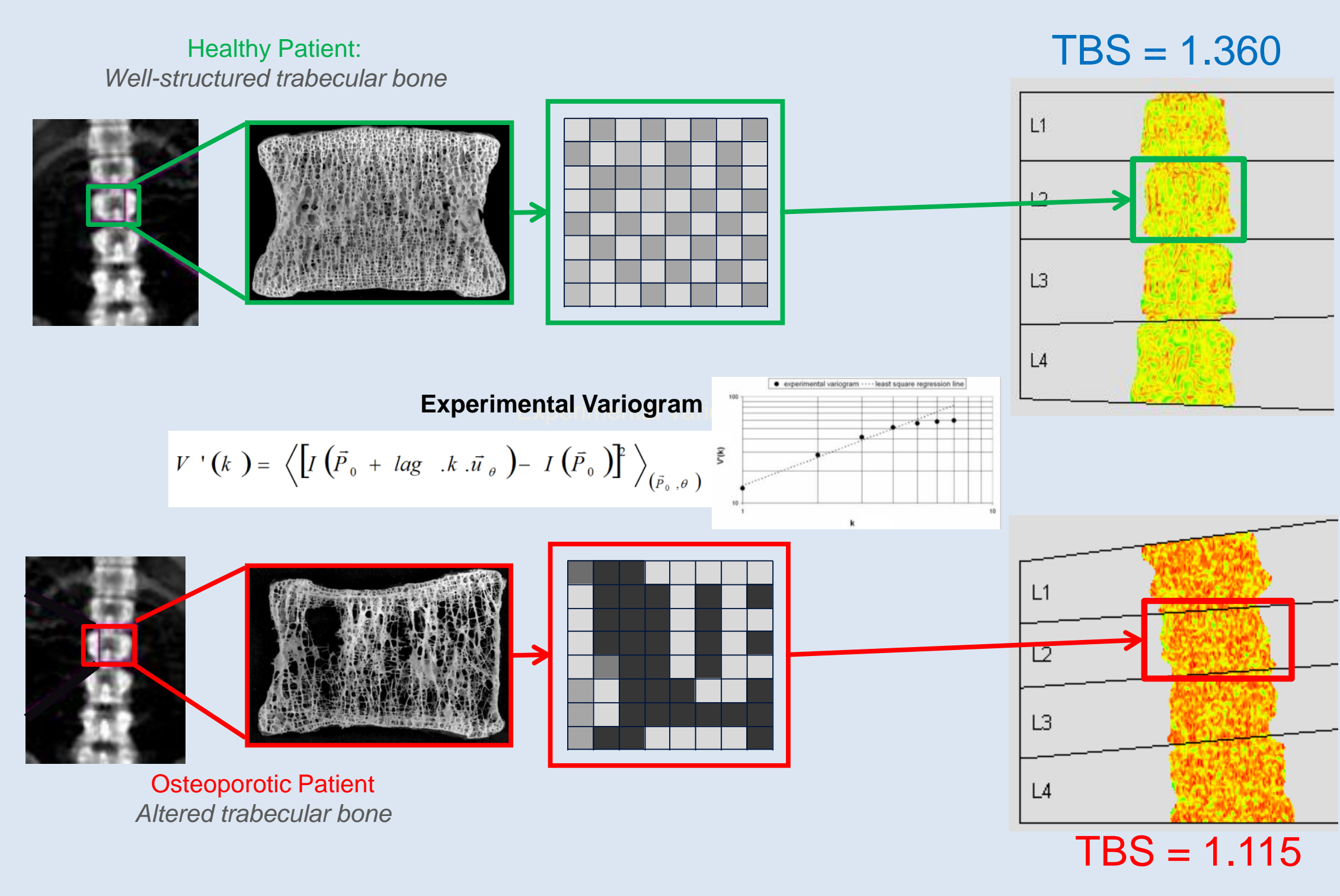

\section{OBJECTIVE:}

\section{PATIENTS AND METHODS}

We included 946 patients (860 women and 89 males) whose were remitted to our facility for evaluate the bone mineral status. In all subjects were assessed the bone mineral density $(\mathrm{d} / \mathrm{cm} 2)$ by DXA (iDXA Healthcare GE, Madison, Wi. Software version 12.3) at lumbar spine (L1-L4) and hip (total hip, femoral neck).

Bone density results were categorized using the T-score for lumbar spine bone mineral density (BMD) into 3 categories according to the WHO recommendations: normal (T-score $>-1$ ), osteopenia (T-score $\leq-1$ and $>-2.5$ ), and osteoporosis (T-score $\leq-2.5$ )

Questionnaires collected information on demographics, personal and family history of major osteoporotic fracture and/or osteoporosis, history of other comorbidities, gynaecologic and obstetric history, and lifestyle, as well as a validated questionnaire on calcium intake.

Patients with vertebral fracture were evaluated by vertebra fracture assessment software on the DXA device and were classified using the Genant semiquantitative approach. TBS was obtained after reanalysis of DXA lumbar spine (L1-L4) scan with TBS iNsight software version
1.8.2.0 (Med-Imaps, Pessac, France). We didn't exclude vertebral fractured to the analysis.

\section{Statistical Analysis:}

Descriptive statistics, including mean and $95 \%$ confidence intervals were estimated for patients with and without vertebral fracture Differences between both groups were analyzed by means of parametric Student $T$ test or non parametric Mann-Whitney test, depending upon distribution normality of the tested parameter. The prevalence of different bone status category, vertebral fracture and osteoporotic fractures (hip, wrist, vertebral or humerus) were calculated. Patients were stratified by TBS tertiles and by BMD Tscore tertiles.

The discriminate value of BMD and TBS (L1-L4) was assessed by determining the area under the receiving operator characteristic (ROC) curve.

All database management and statistical analyses were performed using the SPSS 16.0 statistical software. A $p<0.05$ was considered statistically significant.

\section{RESULTS}

According to the WHO classification, the $31.61 \%$ were classified as normal subjects by BMD.

Of 946 patients studied, 63 have been had at least a vertebral fracture or combined with another fracture (with wrist: $0.85 \%$ to $0.11 \%$ hip), representing a total prevalence of $6.67 \%$ vertebral fractures. Fracture patients had significant lower bone mineral density and TBS.

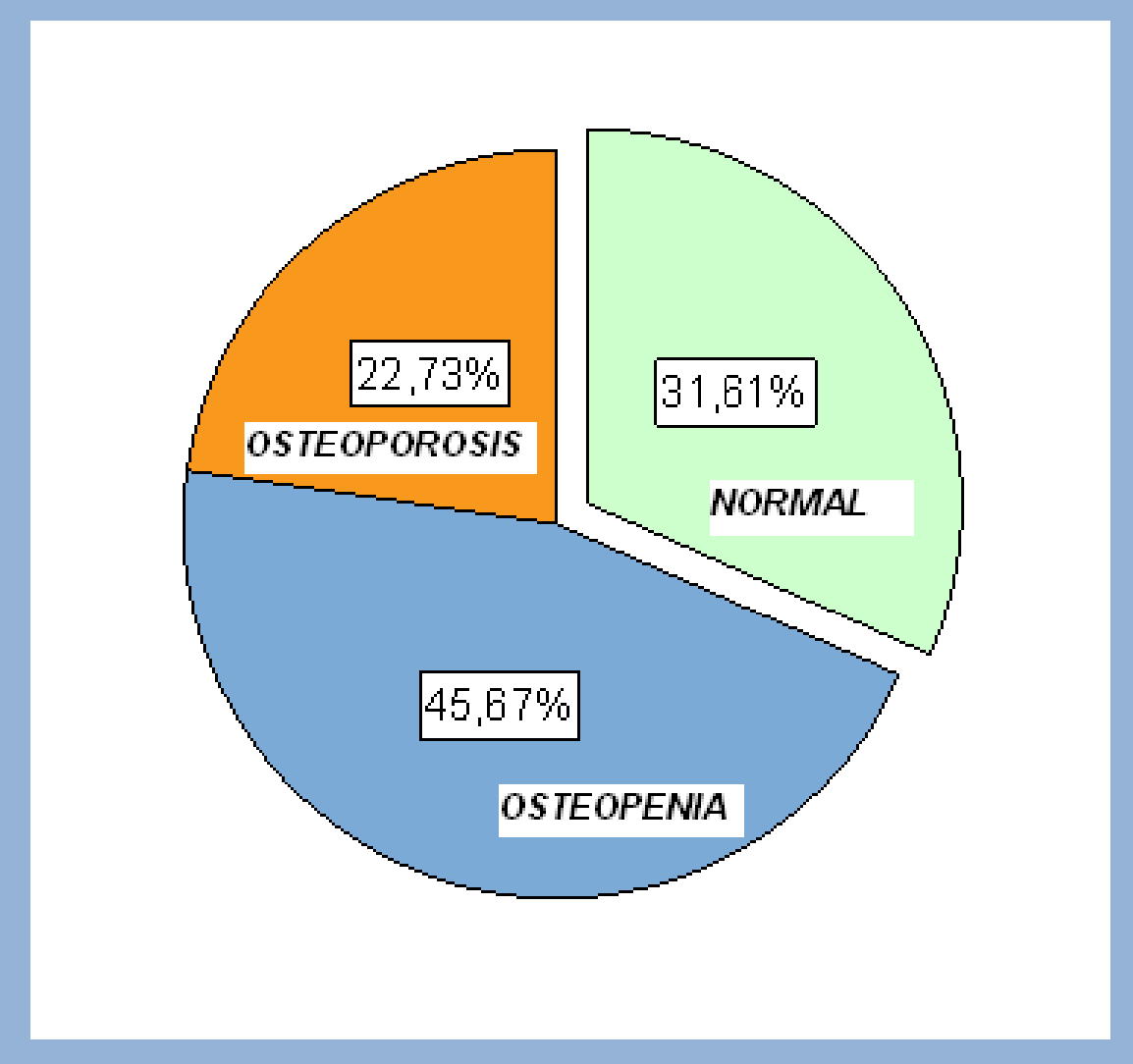

The Pearson correlation indicates a relationship between the two parameters modest claim that bone is oriented evaluation from different perspectives

Pearson Correlation :

TBS (L1-L4) vs DMO g/cm² (L1-L4) $\quad r=0.339$

TBS (L1-L4) vs T-score (L1-L4) r $\quad \mathrm{r}=0.353$

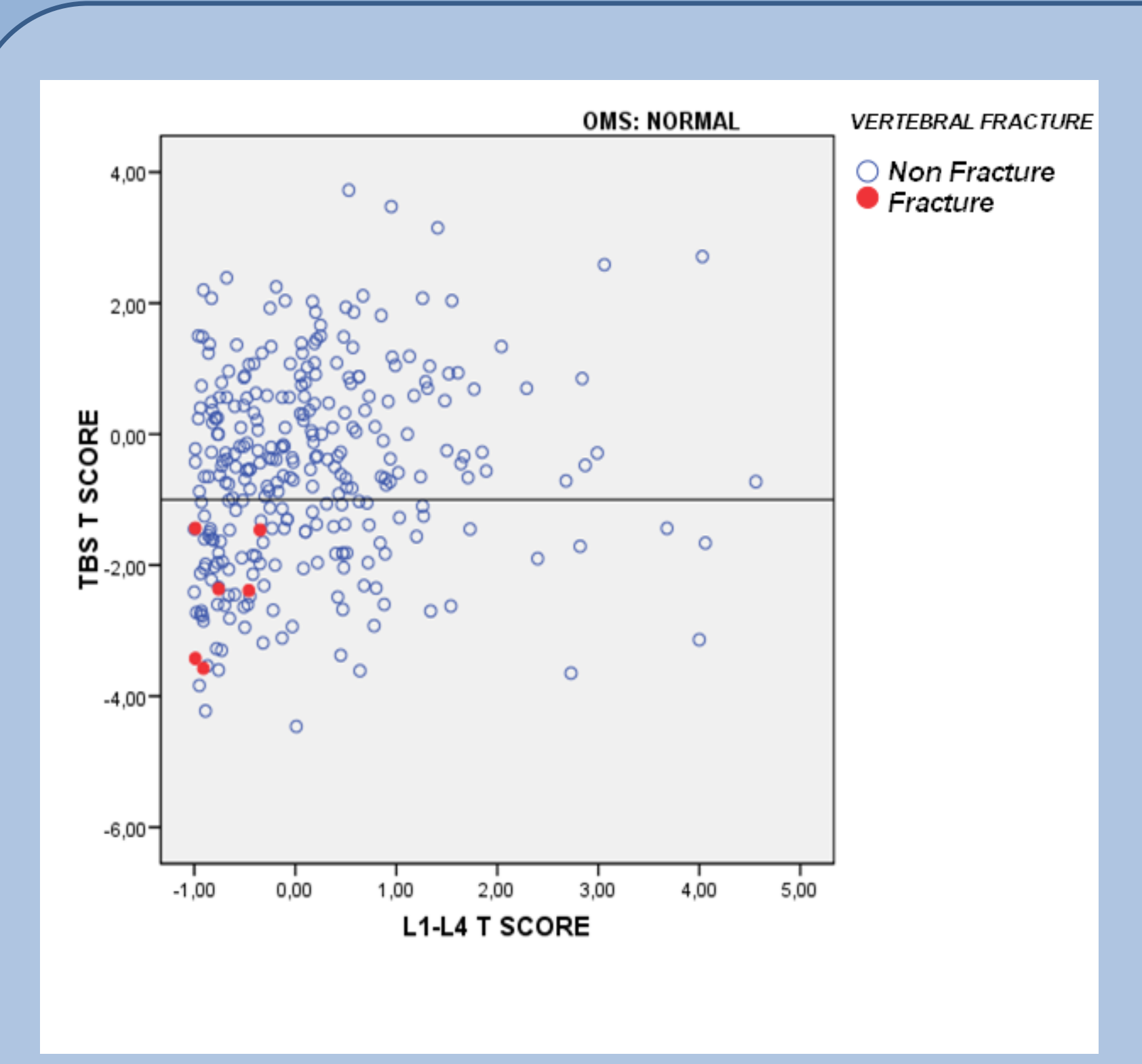

TBS identifies all patients with fragility fractures with normal BMD

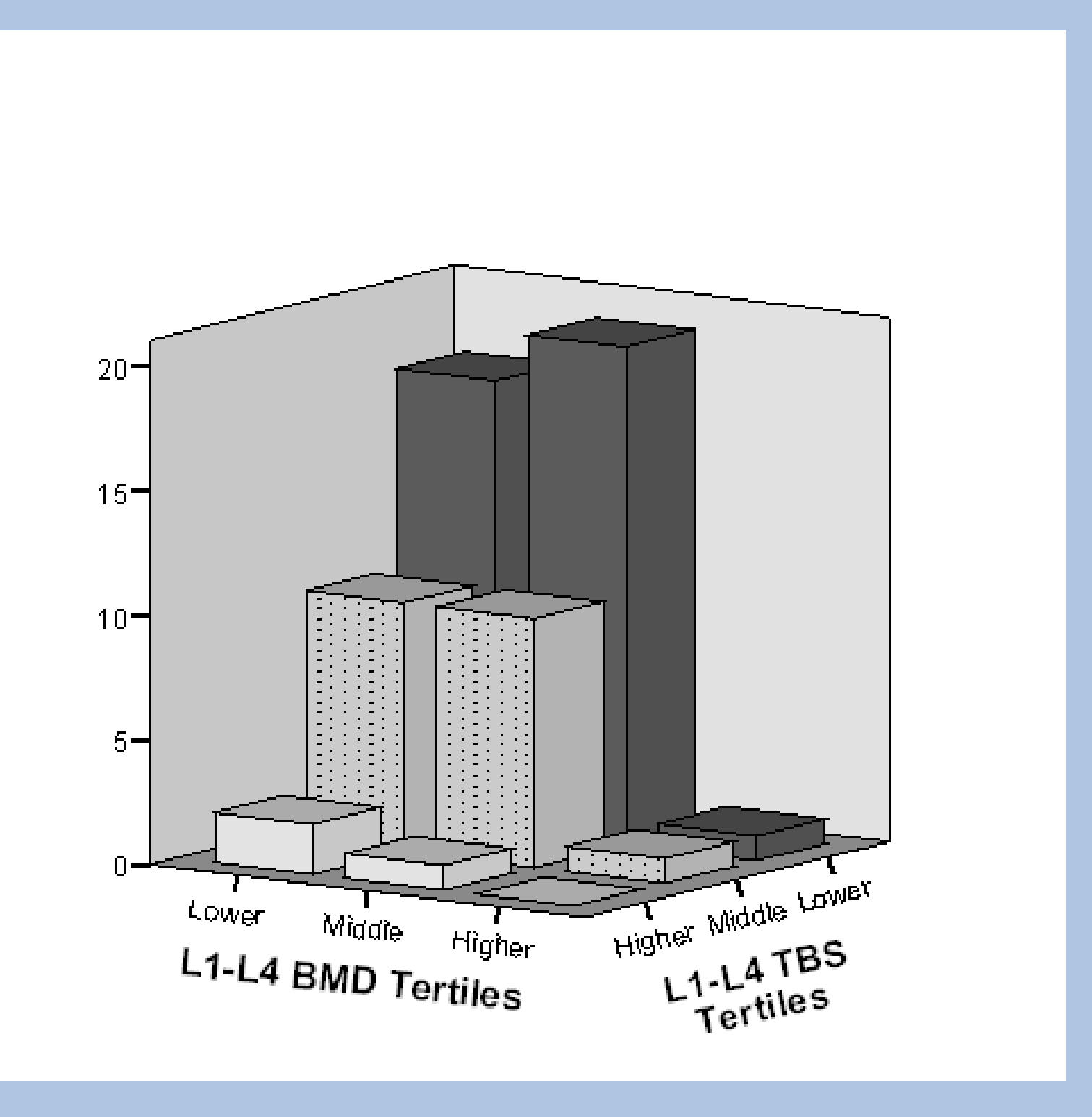

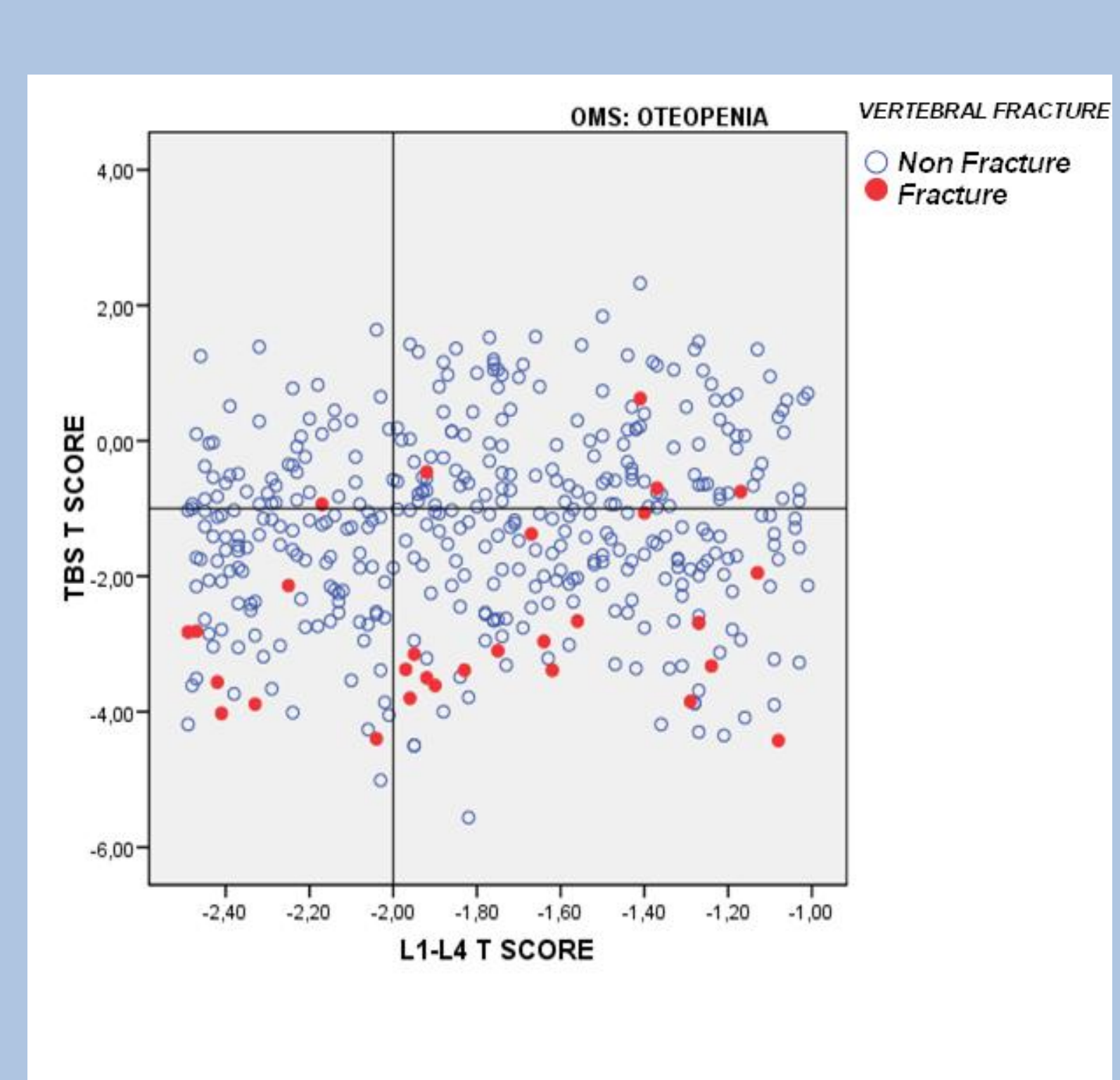

TBS identifies the $82 \%$ of patients with fragility fractures osteopenic by their BMD

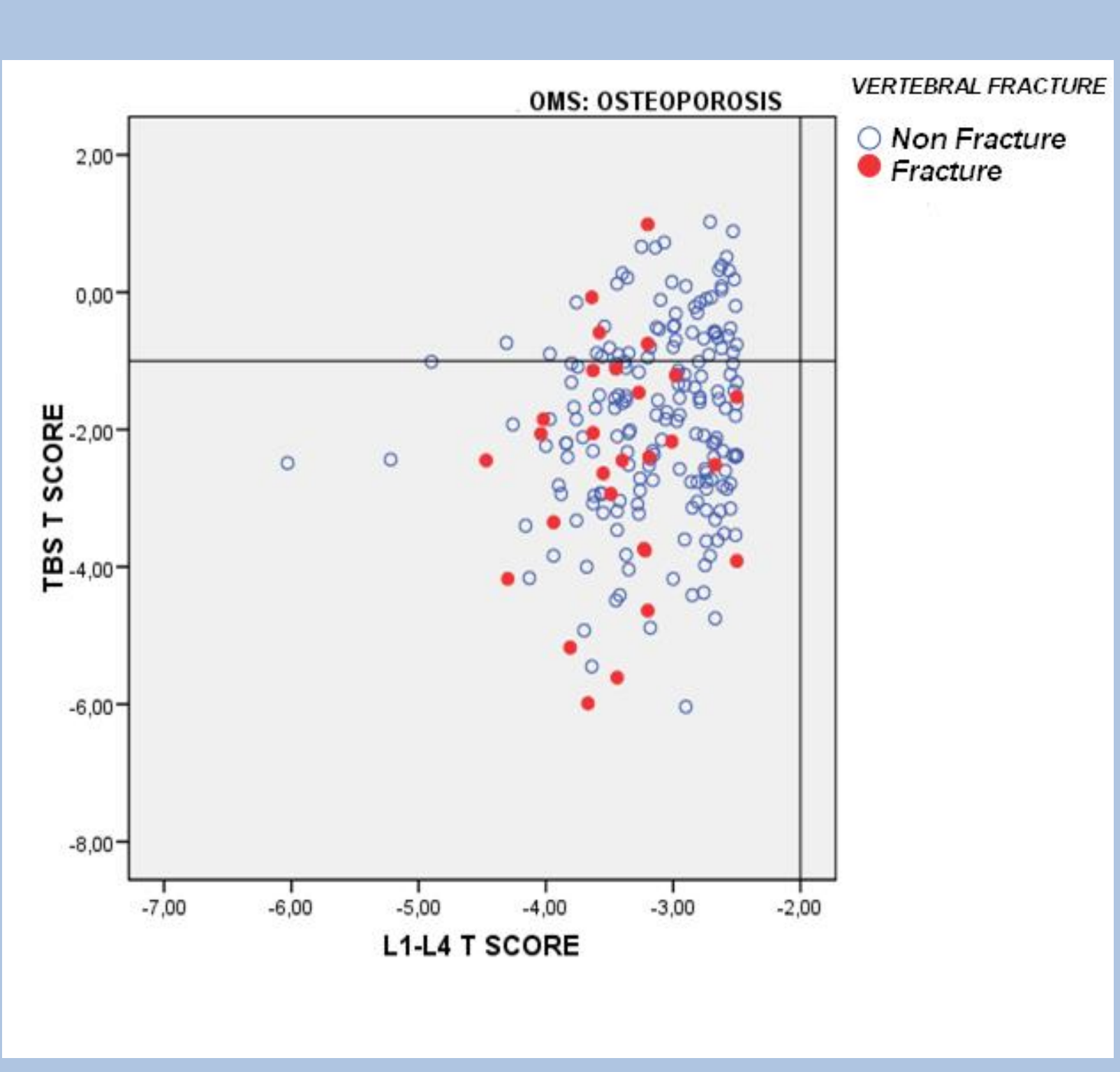

TBS said structural alterations in $85 \%$ of patients with fragility fractures their BMD
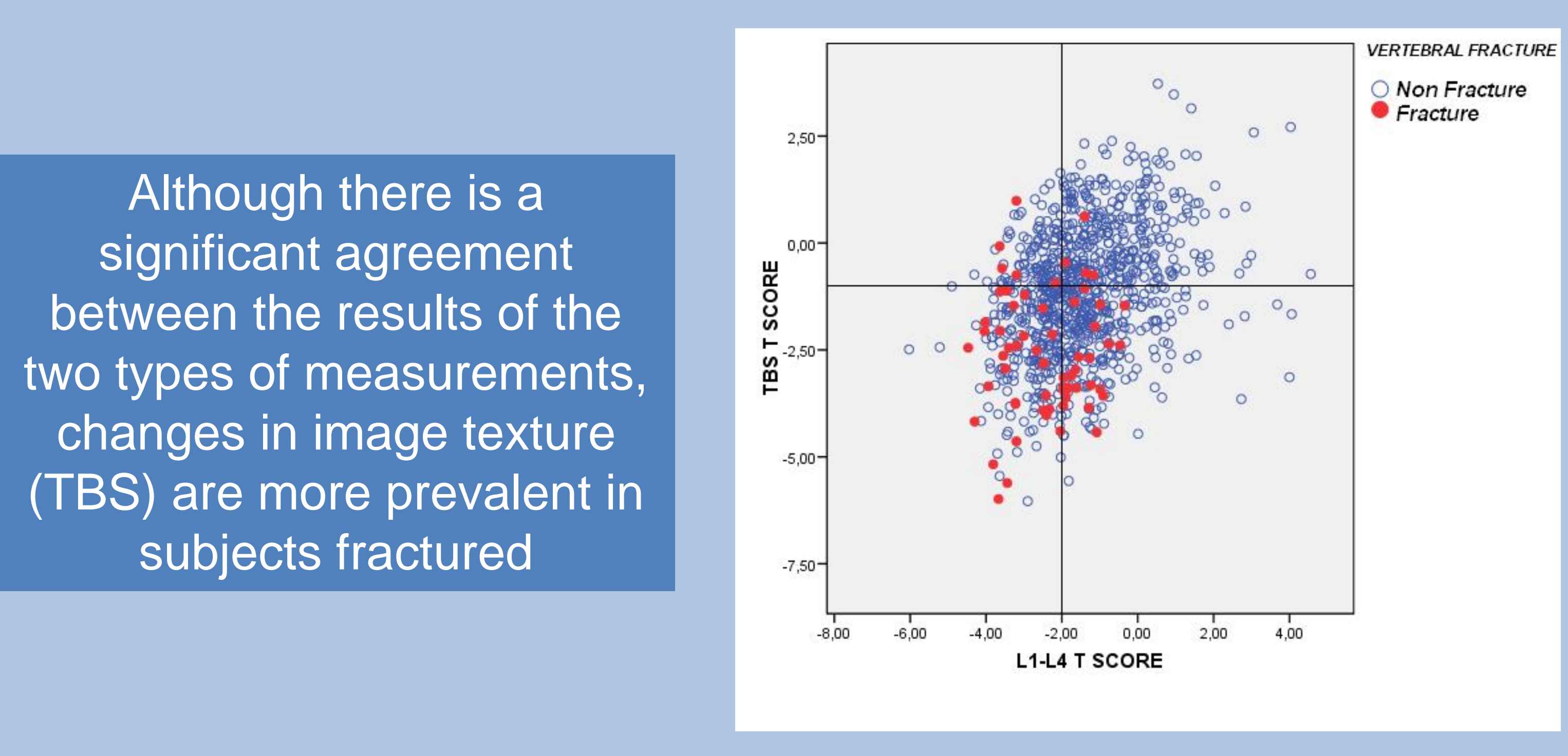
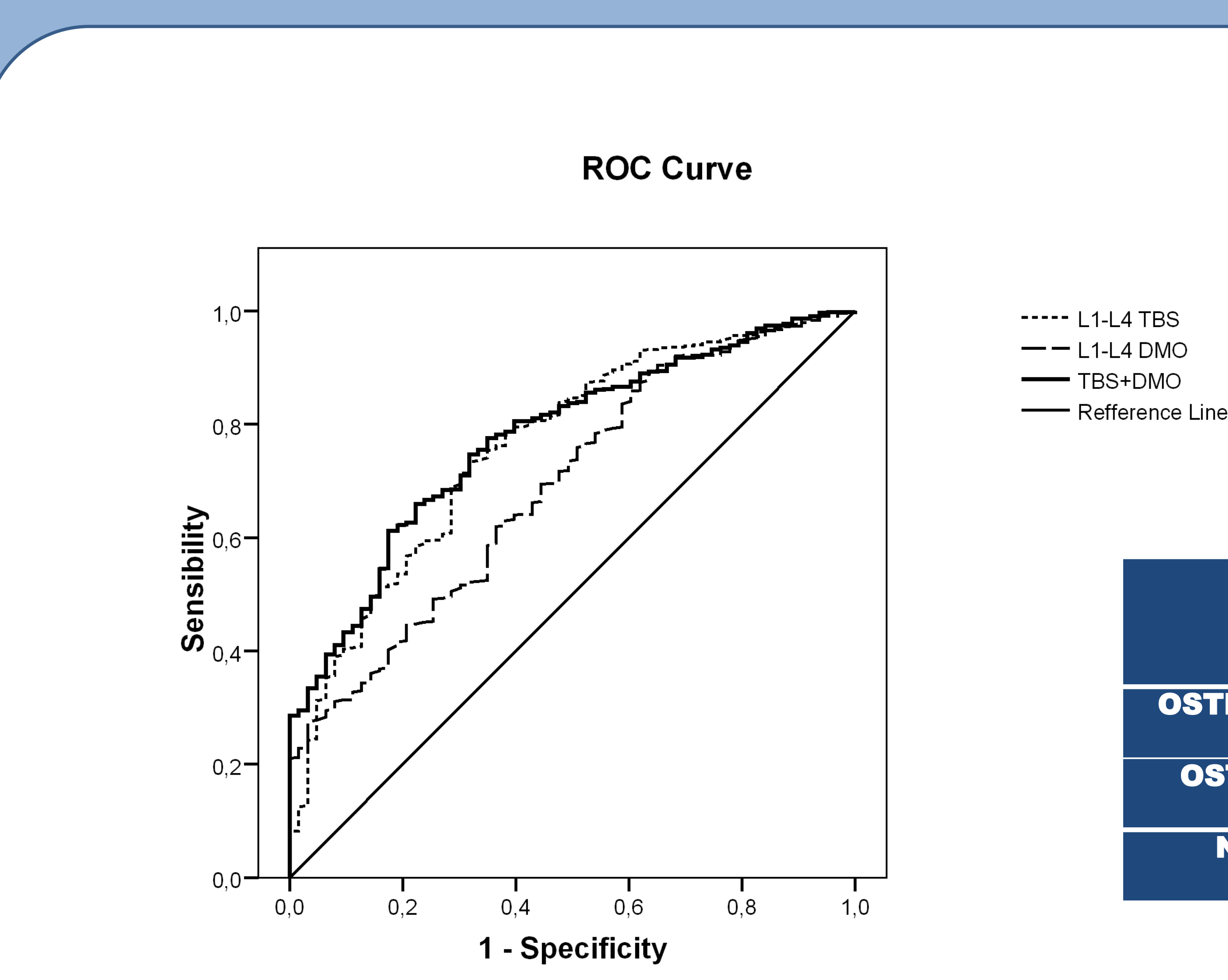

Major TBS clinical added values are in the osteopenic and normal T-score areas

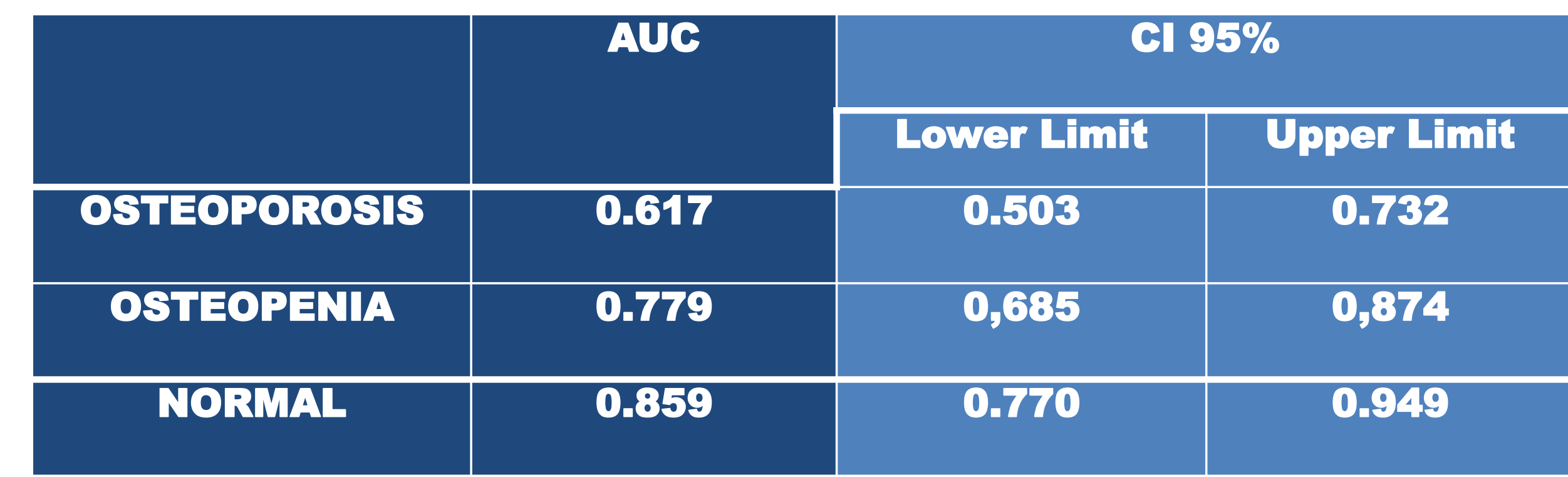

$$
\begin{array}{|c|c|c|c|} 
& & \multicolumn{2}{|c|}{\text { C1 95\% }} \\
\hline \text { L1-L4 TBS } & \text { Area } & \text { Lower Limit } & \text { Upper Limit } \\
\hline \text { L1-L4 DMO } & 0.690 & 0.701 & 0.821 \\
\hline \text { TBS+DMO } & 0.775 & 0.726 & 0.755 \\
\hline
\end{array}
$$

\section{CONCLUSIONS}

- TBS bone quality parameters measured non-dependent, but related to BMD.

- TBS software is a complement to DXA BMD measurements, which provides a significant improvement in diagnostic performance.

- The segment of the population classified densitometry situation "Normal" or "osteopenia" is one that has a larger display to complement your exploration with TBS Assessment. 